\title{
Computational Aspects of Incomplete Gamma Functions With Large Complex Parameters
}

\author{
N.M. Temme*
}

\section{Dedicated to Walter Gautschi on the occasion of his 65th birthday}

Abstract. The incomplete gamma functions are defined by the integrals

$$
\gamma(a, z)=\int_{0}^{z} t^{a-1} e^{-t} d t, \quad \Gamma(a, z)=\int_{z}^{\infty} t^{a-1} e^{-t} d t
$$

where $\Re a>0$. We present several series representations and other relations which can be used for numerical evaluation of these functions. In particular, asymptotic representations for large complex values of the parameter $a$ and $z$ are considered. The results of numerical tests and the set-up of an algorithm will appear in a future paper.

\section{INTRODUCTION}

The incomplete gamma functions are defined by

$$
\gamma(a, z)=\int_{0}^{z} t^{a-1} e^{-t} d t, \quad \Gamma(a, z)=\int_{z}^{\infty} t^{a-1} e^{-t} d t
$$

For $\gamma(a, z)$ we assume the condition $\Re a>0$, and with respect to $z$ we assume $|\arg z|<\pi$. Analytical continuation with respect to both parameters is discussed below. It is useful to have the normalizations

$$
P(a, z)=\frac{\gamma(a, z)}{\Gamma(a)}, \quad Q(a, z)=\frac{\Gamma(a, z)}{\Gamma(a)},
$$

of which the sum equals unity. $P$ and $Q$ are in particular used in statistics and probability theory in connection with the gamma distribution.

The point $z=0$ is a singularity for the incomplete gamma functions, except when $a=0,1,2,3, \ldots$. The singularity at $z=0$ becomes apparent in the representation

$$
\gamma^{*}(a, z)=z^{-a} P(a, z)=\frac{z^{-a}}{\Gamma(a)} \gamma(a, z) ;
$$

$\gamma^{*}(a, z)$ is an entire function of $z$ and $a$.

\footnotetext{
*CWI, P.O. Box 94079, 1090 GB Amsterdam, The Netherlands; nicot@cwi.nl
} 
The following recurrence relations are easily derived from the definitions in (1.1):

$$
\gamma(a+1, z)=a \gamma(a, z)-z^{a} e^{-z}, \quad \Gamma(a+1, z)=a \gamma(a, z)+z^{a} e^{-z} .
$$

Some care is needed when the relations in (1.4) are used in numerical computations. When the parameters are positive, the first relation is unstable.

When $a$ is a non-negative integer the incomplete gamma functions are very simple:

$$
\begin{aligned}
& \gamma(n+1, z)=n !\left[1-e^{-z} e_{n}(z)\right] \quad n=0,1,2, \ldots, \\
& \Gamma(n+1, z)=n ! e^{-z} e_{n}(z) .
\end{aligned}
$$

in which $e_{n}(z)$ is the first part of the Taylor series of the exponential function:

$$
e_{n}(z)=\sum_{m=0}^{n} \frac{z^{m}}{m !}, \quad n=0,1,2, \ldots .
$$

The relations with the Kummer functions (confluent hypergeometric functions) are as follows:

$$
\begin{aligned}
\gamma(a, z) & =a^{-1} z^{a} e^{-z} M(1, a+1, z) \\
& =a^{-1} z^{a} M(a, a+1,-z), \\
\gamma^{*}(a, z) & =\frac{e^{-z}}{\Gamma(a+1)} M(1, a+1, z) \\
& =\frac{1}{\Gamma(a+1)} M(a, a+1,-z), \\
\Gamma(a, z) & =z^{a} e^{-z} U(1, a+1, z) \\
& =e^{-z} U(1-a, 1-a, z) .
\end{aligned}
$$

Important series expansions are

$$
\gamma(a, z)=e^{-z} \sum_{n=0}^{\infty} \frac{z^{a+n}}{(a)_{n+1}}=\sum_{n=0}^{\infty} \frac{(-1)^{n}}{n !} \frac{z^{a+n}}{a+n} .
$$

The series are convergent for each complex $a$ and $z$, with the exception of $a=$ $0,-1,-2, \ldots$ Speed of convergence of the first series depends on the ratio $|z / a|$; when $|z / a|<1$ the convergence is quite fast.

When $z \gg a$, we can use an asymptotic expansion of $\Gamma(a, z)$. This expansion follows from the representation

$$
\Gamma(a, z)=z^{a} e^{-z} \int_{0}^{\infty}(t+1)^{a-1} e^{-z t} d t,
$$

and integrating by parts. We obtain for $N=0,1,2, \ldots$

$$
\Gamma(a, z)=z^{a-1} e^{-z}\left[\sum_{n=0}^{N-1^{\prime}} \frac{(-1)^{n}(1-a)_{n}}{z^{n}}+\theta_{N} \frac{(-1)^{N}(1-a)_{N}}{z^{N}}\right],
$$


(when $N=0$ the sum is empty). The quantity $\theta_{N}$ is the remainder:

$$
\theta_{N}=z \int_{0}^{\infty}(t+1)^{a-N-1} e^{-z t} d t
$$

which in fact is an incomplete gamma function (see (1.8)):

$$
\theta_{N}=z^{N+1-a} e^{z} \Gamma(a-N, z)
$$

This relation also follows from repeated application of the recurrence relation in (1.4).

For positive values of the parameters we can obtain an interesting estimate for $\theta_{N}$. We need the condition $z>a-N$. Then we write

$$
\theta_{N}=z^{N+1-a} e^{z} \int_{z}^{\infty} t^{a-N-1} e^{-t} d t
$$

and next we integrate with respect to $u=t-(a-N) \ln t$. We obtain

$$
\theta_{N}=z^{N+1-a} e^{z} \int_{z_{0}}^{\infty} \frac{e^{-u}}{t-(a-N)} d u<\frac{z}{z-(a-N)}
$$

in which $z_{0}=z-(a-N) \ln z$.

Expansion (1.9) is re-considered in Olver (1991), in particular in connection with the Stokes phenomenon and the role of exponentially small terms in this expansion. Expansion (1.9) is valid in the sector $|\arg z|<\frac{3}{2} \pi$, with $a \in \mathbb{C}$ fixed. Olver investigates the expansion at the Stokes line $|\arg z|= \pm \pi$ and gives a uniform asymptotic expansion of $\Gamma(a, z)$ which is valid in a much larger $z$-domain. In fact Olver gives an expansion of the remainder $\theta_{N}$ for large $|z|$ and $N$. Considering (1.10) we observe that this problem is related with the uniform expansion of the incomplete gamma function with both parameters large given in (5.2) and (5.3).

Another important tool for numerical computations is Legendre's continued fraction:

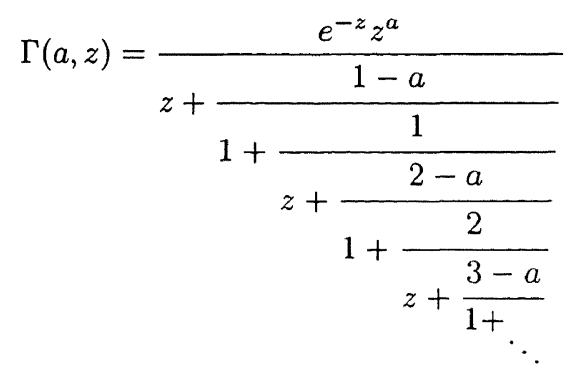

This expansion converges for all $z \neq 0,|\arg z|<\pi$ and any complex value of $a$. It is a splendid addition to the asymptotic expansion (1.9). The continued fraction 
converges better as the ratio $|z / a|$ increases. An extensive discussion on the use of (1.11) and other continued fractions for the incomplete gamma functions is given in Jones \& Thron (1985).

In the literature many articles on the evaluation of the incomplete gamma functions can be found. It is outside the scope of the present paper to give a full overview. In the recent survey Lozier \& Olver (1994) references to many papers and software are given.

Apart from the series expansions, continued fractions, Chebyshev and Padé expansions mentioned in the present paper, one can use other numerical techniques. For instance, in Allasia \& Besenghi (1987) numerical quadrature is used.

The incomplete gamma functions occur in the literature also as generalized exponential integrals, which are usually defined by

$$
E_{\nu}(z)=\int_{1}^{\infty} t^{-\nu} e^{-z t} d t, \quad \nu \in \mathbb{C}, \quad \Re z>0 .
$$

The relation with $\Gamma(a, z)$ is $E_{\nu}(z)=z^{\nu-1} \Gamma(1-\nu, z)$. In a set of papers Chiccoli et al. (1987-1990) the generalized exponential integrals are extensively discussed, with emphasis on real $\nu$ and positive $z$.

\section{ALGORITHMS FOR REAL PARAMETERS}

When $a$ and $z=x$ are positive one usually concentrates on the normalized functions $P(a, x)$ and $Q(a, x)$. First the smaller of these two is computed; the relation $P(a, x)+Q(a, x)=1$ gives the other value. We have the following rule (with slight corrections for small values of $x$ and $a)$ :

$0 \leq a \leq x$ : first compute $Q$, then $P=1-Q$;

$0 \leq x<a$ : first compute $P$, then $Q=1-P$.

This splitting of the $(a, x)$-domain follows from the asymptotic relation

$$
P(a, a), Q(a, a) \sim \frac{1}{2}, \quad \text { as } \quad a \rightarrow \infty .
$$

The method for $Q$ is usually based on Legendre's continued fraction (1.11), say when $x>1$. When $x \gg a$ the asymptotic expansion (1.9) may be used for $Q$. The computation of $P$ may be based on the first series occurring in (1.7), that is,

$$
P(a, x)=x^{a} e^{-x} \sum_{n=0}^{\infty} \frac{x^{n}}{\Gamma(a+n+1)} .
$$

For large values of the parameters $x$ and $a$, especially when the parameters are nearly equal, the expansions for computing $P$ and $Q$ converge slowly. In that case an algorithm based on a uniform asymptotic expansion can be used; see $\S 5$. References for software for the real case are DiDonato \& Morris (1986), Gautschi (1979) and Temme (1994). 


\section{OTHER SERIES EXPANSIONS}

All expansions in this section give excellent approximations for restricted domains of the parameters, although the expansions are all valid in large domains of the complex plane. It is quite difficult to give a priori information on the applicability of the expansions in efficient and reliable numerical algorithms. As a rule, the expansions for $\gamma(a, z)$ should be used in a $z$-domain around the origin, while the expansions for $\Gamma(a, z)$ are of interest in a $z$-domain containing the point $\infty$.

\section{Chebyshev expansions}

Let $T_{n}(x)$ denote the Chebyshev polynomial (that is, $T_{n}(\cos \theta)=\cos n \theta$ ) and let $T_{n}^{*}(x)=T_{n}(2 x-1)$ be the shifted Chebyshev polynomial. From expansions of the Kummer function $M(a, c, z)={ }_{1} F_{1}(a, c, z)$ we derive, see Luke (1969, II, p. 35),

$$
\gamma^{*}(a, z x)=\frac{e^{-z x}}{\Gamma(a+1)} \sum_{n=0}^{\infty} C_{n}(z) T_{n}^{*}(x), \quad 0 \leq x \leq 1 .
$$

The coefficients can be computed by a backward recurrence algorithm using the relation

$$
\frac{2 C_{n}(z)}{\varepsilon_{n}}=[-1+4(n+1+a) / z] C_{n+1}(z)+[1+4(n+2-a) / z] C_{n+2}(z)+C_{n+3}(z),
$$

where $\varepsilon_{0}=1, \varepsilon_{n}=2$ if $n \geq 1$.

From expansions of the Kummer function $U(a, c, z)=\psi(a, c, z)$ we derive, see Luke (1969, II, p. 25),

$$
\Gamma(a, \omega z)=(\omega z)^{a-1} e^{-\omega z} \sum_{n=0}^{\infty} C_{n}(z) T_{n}^{*}(1 / \omega), \quad|\arg z|<\frac{3}{2} \pi, \quad 1 \leq \omega \leq \infty
$$

provided that $z \neq 0, a \neq 1,2,3, \ldots$. When $a$ is a positive integer the incomplete gamma functions reduce to elementary functions; see (1.4). We have the recurrence relation

$$
\begin{aligned}
\frac{2(n+1-a)}{\varepsilon_{n}} C_{n}(z)= & -(3 n+4+4 z-a) C_{n+1}(z)-(3 n+5-4 z+a) C_{n+2}(z) \\
& -(n+2+a) C_{n+3}(z),
\end{aligned}
$$

$n=0,1,2, \ldots$ Luke gives the following estimate of these coefficients:

$$
C_{n}(z)=\frac{4(-1)^{n} \sqrt{\pi} n^{-2 a / 3} z^{(3-2 a) / 6}}{\sqrt{3} \Gamma(1-a)} e^{-3 n^{2 / 3} z^{1 / 3}}\left[1+\mathcal{O}\left(n^{-1 / 3}\right],\right.
$$

$|\arg z|<3 \pi / 2$, from which follows excellent convergence, in particular when $z$ is large. The speed of convergence of the backward recurrence algorithm deteriorates, 
however, when $z$ approaches the negative real axis. Also, large complex values of $a$ may have a bad influence on the rate of convergence of both the series expansion and the recurrence algorithms. When comparing the Chebyshev expansion with the continued fraction we found that the continued fraction is easier to implement, since in that case a forward recurrence relation for evaluating the continued fraction can be used. See Gautschi (1979), where the continued fraction is evaluated as a series. Controlling the error term is easier then, although a reliable stopping criterion for obtaining a certain accuracy is difficult to obtain for general complex values of the parameters.

\section{Padé approximations for $\gamma^{*}(a, z)$}

From Luke (1969, II, p. 190) we obtain

$$
\gamma^{*}(a,-z)=\frac{e^{z}}{\Gamma(a+1)}\left[\frac{A_{n}}{B_{n}}+V_{n}\right]
$$

where

$$
A_{0}=B_{0}=1, \quad A_{1}=1-\frac{z}{(a+1)(a+2)}, \quad B_{1}=1+\frac{z}{a+2},
$$

and both $A_{n}$ and $B_{n}$ satisfy the recurrence formula

$$
\begin{aligned}
B_{n+1} & =\left[1+\frac{a z}{(2 n+a)(2 n+a+2)}\right] B_{n}+\frac{n(n+a)}{(2 n+a-1)(2 n+a)^{2}(2 n+a+1)} B_{n-1}, \\
V_{n} & =\frac{(-1)^{n+1} \pi \Gamma(a+1) n ! \Gamma(n+a+1) z^{2 n+1} e^{\chi}}{2^{4 n+2 a}(2 n+a+1)\left\{\Gamma[n+(a+1) / 2] \Gamma[(n+(a+2) / 2]\}^{2}\right.}\left[1+\mathcal{O}\left(n^{-3}\right)\right],
\end{aligned}
$$

as $n \rightarrow \infty$ with $z$ and $a$ fixed, and $\chi=-z+z(z+4 a) /[4(2 n+a+1)]$.

\section{Bessel function expansions}

We have

$$
\begin{aligned}
\gamma^{*}(a, z) & =\frac{e^{-\frac{1}{2} z}\left(\frac{1}{4} z\right)^{-a+\frac{1}{2}} \Gamma\left(a-\frac{1}{2}\right)}{\Gamma(a+1)} \sum_{n=0}^{\infty} \frac{(2 a-1)_{n}(a-1)_{n}(-1)^{n}}{n !(a+1)_{n}} I_{a-\frac{1}{2}+n}\left(\frac{1}{2} z\right), \\
\gamma^{*}(a,-z) & =\frac{2 \sqrt{\pi} e^{\frac{1}{2} z}}{\sqrt{z} \Gamma(a+1)} \sum_{n=0}^{\infty} \frac{(1-a)_{n}(-1)^{n}}{(a+1)_{n}} I_{n+\frac{1}{2}}\left(\frac{1}{2} z\right),
\end{aligned}
$$

where in both cases $a \neq-1,-2,-3, \ldots$ More expansions of this kind are given in Luke (1969, II, p. 48). 


\section{Tricomi's expansions}

Let

$$
E_{\nu}(z):=z^{-\frac{1}{2} \nu} J_{\nu}(2 \sqrt{z}),
$$

the Bessel function without its algebraic singularity; $E_{\nu}(z)$ is an entire function of both $z$ and $\nu$, with $E_{\nu}(0)=1 / \Gamma(\nu+1)$.

In Luke (1969, I, p. 129) Tricomi's expansions are cast into the form:

$$
\frac{e^{-h z}}{\Gamma(c)} F(a, c, z)=\sum_{n=0}^{\infty} C_{n} z^{n} E_{m+c-1}(\omega z), \quad|z|<\infty,
$$

where $C_{n}$ depends only on the parameters $a, c, h$ and $\omega$, and are determined by the generating function

$$
e^{\omega z}(1+h z)^{a-c}[1+(h-1) z]^{-a}=\sum_{n=0}^{\infty} C_{n} z^{n} .
$$

That is, $C_{0}=1, C_{1}=\omega+a-c h, 2 C_{2}=C_{1}^{2}+c h^{2}-2 h a+a$, and we have the recurrence formula

$$
\begin{aligned}
(n+1) C_{n+1} & =\left[C_{1}-(2 h-1) n\right] C_{n}+[\omega(2 h-1)-h(h-1)(n+c-1)] C_{n-1} \\
& +\omega h(h-1) C_{n-2}
\end{aligned}
$$

where $n=2,3, \ldots$. Here $\omega$ and $h$ are free parameters. We interpret this expansion for the case of the incomplete gamma functions:

$$
\gamma^{*}(a, z)=e^{(h-1) z} \sum_{n=0}^{\infty} A_{n} z^{n} E_{n+a}(\omega z),
$$

with generating function

$$
e^{\omega z}(1+h z)^{-a}[1+(h-1) z]^{-1}=\sum_{n=0}^{\infty} A_{n} z^{n} .
$$

That is, $A_{0}=1, A_{1}=\omega+1-(a+1) h, 2 A_{2}=A_{1}^{2}+(a+1) h^{2}-2 h+1$ with recurrence formula

$$
(n+1) A_{n+1}=\left[A_{1}-(2 h-1) n\right] A_{n}+[\omega(2 h-1)-h(h-1)(n+a)] A_{n-1}+\omega h(h-1) A_{n-2} .
$$

Tricomi considered $\omega$ as function of $a$ and $z$ in order to get a series that has asymptotic properties as $a \rightarrow \infty$; see Luke (1969, I, p. 129) for more details. 


\section{UNIFORM ASYMPTOTIC EXPANSIONS}

We give expansions for $\Gamma(-a, z)$ and $\gamma^{*}(a,-z)$ which are suitable when both parameters $a$ and $z$ are large. Let $\lambda=z / a$; in this section we assume that $\lambda+1$ is bounded away from zero. From Gautschi (1959) we obtain

$$
\begin{aligned}
z^{a} \Gamma(-a, z) & =\int_{1}^{\infty} e^{-a(\lambda t+\ln t)} g_{0}(t) \frac{d t}{t} \\
& =-\frac{1}{a} \int_{1}^{\infty} \frac{g_{0}(t)}{1+\lambda t} d e^{-a(\lambda t+\ln t)} \\
& =\frac{e^{-z}}{a} \frac{1}{\lambda+1}+\frac{1}{a} \int_{1}^{\infty} e^{-a(\lambda t+\ln t)} g_{1}(t) \frac{d t}{t},
\end{aligned}
$$

where $g_{0}(t)=1, g_{1}(t)=t \frac{d}{d t}\left[g_{0}(t) /(1+\lambda t)\right]$. Continuing this we obtain

$$
z^{a} \Gamma(-a, z)=\frac{e^{-z}}{a} \sum_{n=0}^{N-1} \frac{C_{n}(\lambda)}{a^{n}}+\frac{R_{N}}{a^{N}}
$$

where, for $n=0,1,2, \ldots$,

$$
R_{n}=\int_{1}^{\infty} e^{-z t} t^{-a-1} g_{n}(t) d t, \quad g_{n}(t)=t \frac{d}{d t} \frac{g_{n-1}(t)}{1+\lambda t},
$$

and the coefficients $C_{n}(\lambda)$ are given by $C_{n}(\lambda)=\frac{g_{n}(1)}{\lambda+1}$. We have the recursion

$$
C_{n}(\lambda)=\frac{\lambda}{\lambda+1} \frac{d}{d \lambda} C_{n-1}(\lambda), \quad C_{0}(\lambda)=\frac{1}{\lambda+1} .
$$

The first few coefficients are

$$
\begin{aligned}
& C_{0}(\lambda)=\frac{1}{\lambda+1}, \quad C_{1}(\lambda)=\frac{-\lambda}{(\lambda+1)^{3}}, \quad C_{2}(\lambda)=\frac{\lambda(2 \lambda-1)}{(\lambda+1)^{5}}, \\
& C_{3}(\lambda)=\frac{-\lambda\left(6 \lambda^{2}-8 \lambda+1\right)}{(\lambda+1)^{7}}, \\
& C_{4}(\lambda)=\frac{\lambda\left(24 \lambda^{3}-58 \lambda^{2}+22 \lambda-1\right)}{(\lambda+1)^{9}} .
\end{aligned}
$$

Gautschi computed (for positive parameters) numerical bounds for the first 7 remainders $R_{n}(\lambda)$ of this expansion. For complex parameters bounds are unavailable. The expansion remains valid when $a$ becomes negative; in fact it holds for $\Gamma(a, z)$ with $a<z$ and and also for complex parameters.

A corresponding expansion follows from the integral

$$
\Gamma(a) \gamma^{*}(a,-z)=\int_{0}^{1} e^{z t} t^{a-1} d t
$$


where $z \in \mathbb{C}$ and $\Re a>0$. Integrating by parts we obtain

$$
\Gamma(a) \gamma^{*}(a,-z)=e^{z} \sum_{n=0}^{N-1}(-1)^{n} \frac{C_{n}(\lambda)}{a^{n+1}}+\frac{S_{N}}{a^{N}},
$$

with the same $C_{n}(\lambda)$ as given above. Both expansions are useful additions to the power series expansions and continued fraction. Just bounding the remainders gives poor information on the applicability of the expansions for general $a$ and $z$.

More analytic insight gives the following. Consider

$$
z^{a} \Gamma(-a, z)=\int_{1}^{\infty} e^{-a(\ln t+\lambda t)} \frac{d t}{t}
$$

Transform: $u=\ln t+\lambda(t-1)$. Then,

$$
z^{a} e^{z} \Gamma(-a, z)=\int_{0}^{\infty} e^{-a u} \frac{d u}{1+\lambda t} .
$$

Similarly,

$$
\Gamma(a) \gamma^{*}(a,-z)=\int_{0}^{1} e^{a(\ln t+\lambda t)} \frac{d t}{t} .
$$

Transform: $v=-\ln t-\lambda(t-1)$. Then,

$$
e^{-z} \Gamma(a) \gamma^{*}(a,-z)=\int_{0}^{\infty} e^{-a v} \frac{d v}{1+\lambda t} .
$$

Now the asymptotic tool is Watson's lemma. Expand

$$
\frac{1}{1+\lambda t}=\sum_{n=0}^{\infty} c_{n}(\lambda) u^{n}=\sum_{n=0}^{\infty}(-1)^{n} c_{n}(\lambda) v^{n}
$$

and substitute this in (4.5) and (4.6). This gives the above expansions (4.1) and (4.4). For both Laplace integrals we need to know the singular points. Clearly, $t=-1 / \lambda$ is the source of the singularities. Corresponding $u, v$-values are:

$$
u_{ \pm}=-\lambda-1-\ln \lambda \pm i \pi, \quad v_{ \pm}=\lambda+1+\ln \lambda \pm i \pi .
$$

When these points are close to the origin, Watson's lemma fails. Observe that $u_{ \pm}, v_{ \pm} \rightarrow 0$ when $\lambda \rightarrow-1$. The radius of convergence of the series in (4.7) equals $\min \left|u_{ \pm}\right|$.

For numerical applications we need a condition like $\left|u_{ \pm}\right|>1$. Next, let $\alpha_{1}:=$ $\arg u_{-}, \alpha_{2}:=\arg u_{+}$and assume that $\alpha_{1}<0, \alpha_{2}>0$, as is the case when starting 
with positive values of $a$ and $z$. Then, according to Olver (1974, p. 114), Watson's lemma applied to (4.5) gives expansion (4.1) holding for $a$-values in the sector

$$
-\alpha_{2}-\frac{1}{2} \pi+\delta \leq \arg a \leq-\alpha_{1}+\frac{1}{2} \pi-\delta,
$$

where $\delta$ is a small positive number. Similarly for $\gamma^{*}(a,-z)$. It is quite difficult to visualize both conditions when the parameters are complex. A numerical verification is not difficult, however.

A first impression of the nature of this expansion follows by considering ratios of successive terms in the expansion. Let $\psi_{n}=C_{n}(\lambda) / a^{n}$. Then we have, when $\lambda$ is large, $\psi_{n+1} / \psi_{n} \sim 1 /(\lambda a)=1 / z$. When $|\lambda| \ll 1$ we have $\psi_{n+1} / \psi_{n} \sim 1 / a$. We see that the scale $\left\{\psi_{n}\right\}$ is a uniform asymptotic scale as both $a$ and $z$ are large, except when $\lambda$ tends to -1 ; large or small values of $\lambda$ do not disturb the asymptotic nature of the scale.

We have derived the expansion for $\gamma^{*}(a,-z)$ using (4.3), under the condition $\Re a>0$, since otherwise representation (4.3) does not converge at the origin. However, by using

$$
\gamma^{*}(a, z)=\frac{\Gamma(1-a)}{2 \pi i} \int_{-1}^{\left(0^{+}\right)} t^{a-1} e^{z t} d t
$$

where the contour starts at $t=-1$ and encircles the origin once in positive direction, with $\arg t=0$ when $t>0$, it is possible to obtain the expansion (4.4) without the restriction $\Re a>0$. The integral in (4.8) is defined for all complex values of $z$ and $a$. The poles of $\Gamma(1-a)$ at $a=1,2,3, \ldots$ are removable singularities in this representation, because the integral vanishes for these integer values of $a$. It is easily verified that

$$
\gamma^{*}(-n, z)=z^{n}, \quad n=0,1,2, \ldots
$$

\section{FURTHER UNIFORM ASYMPTOTIC EXPANSIONS}

Let $\eta$ be the real number defined by

$$
\frac{1}{2} \eta^{2}=\lambda-1-\ln \lambda, \quad \lambda>0, \quad \operatorname{sign}(\eta)=\operatorname{sign}(\lambda-1) .
$$

Extend $\lambda$ and $\eta$ to complex values. Then we write, with $\lambda=z / a$,

$$
\begin{aligned}
& Q(a, z)=\frac{\Gamma(a, z)}{\Gamma(a)}=\frac{1}{2} \operatorname{erfc}(\eta \sqrt{a / 2})+R_{a}(\eta), \\
& P(a, z)=\frac{\gamma(a, z)}{\Gamma(a)}=\frac{1}{2} \operatorname{erfc}(-\eta \sqrt{a / 2})-R_{a}(\eta) .
\end{aligned}
$$


The error functions are the dominant terms, that describe the transition at $a=z$. Observe that the property $P+Q=1$ is reflected in the error functions, since $\operatorname{erfc} z+\operatorname{erfc}(-z)=2$, and in having the same term $R_{a}(\eta)$. We have

$$
R_{a}(\eta) \sim \frac{e^{-\frac{1}{2} a \eta^{2}}}{\sqrt{2 \pi a}} \sum_{n=0}^{\infty} \frac{C_{n}(\eta)}{a^{n}}, \quad \text { as } \quad a \rightarrow \infty,
$$

uniformly with respect to $\eta$; in particular, $|\eta|$ may be small. Further investigations are needed to obtain the $(a, z)$-domain in which the above expansion can be used for numerical algorithms. For positive parameters, see Temme (1987), (1994).

We need a representation and expansion like (5.2), (5.3) in the left half planes. From Tricomi (1950) we have, with $\lambda=z / a$,

$$
\gamma^{*}(-a,-z)=\frac{z^{a} \sin \pi a \Gamma(a+1)}{\pi} \int_{z}^{a} e^{t} \frac{d t}{t^{a+1}}+\lambda^{a} \gamma^{*}(-a,-a) .
$$

This can be proved by using series expansions, or the fact that $y(z)=$ $z^{-a} \gamma^{*}(-a,-z)$ satisfies the equation $z y^{\prime \prime}+(a+1-z) y^{\prime}=0$.

The integral can be transformed into

$$
G_{a}(\eta)=e^{-\frac{1}{2} \eta^{2}} \int_{0}^{\eta} e^{\frac{1}{2} a \zeta^{2}} f(\zeta) d \zeta,
$$

where $\eta$ is given in (5.1). $G_{a}(\eta)$ reduces to Dawson's integral (error function with purely imaginary argument) if $f=1$; Dawson's integral is the main approximant in the uniform expansion of $G_{a}(\eta)$. Furthermore a series like the one in (5.3) plays a role. Representation (5.4) and the uniform asymptotic expansion containing Dawson's integral have to be investigated for numerical applications. The method developed in Temme (1987) may be applicable to this new expansion.

Acknowledgment. The author wishes to thank the referee for suggesting several new references.

\section{REFERENCES}

[1] Allasia G., Besenghi R. Numerical calculation of incomplete gamma functions by the trapezoidal rule. Numer. Math., 50:419-428, 1987.

[2] Chiccoli C., Lorenzutta S., Maino G. A numerical method for generalized exponential integrals. Comput. Math. Applic., 14:261-268, 1987.

[3] Chiccoli C., Lorenzutta S., Maino G. On the evaluation of generalized exponential integrals $E_{\nu}(x)$. J. Comput. Phys., 78:278-286, 1988.

[4] Chiccoli C., Lorenzutta S., Maino G. Calculation of exponential integrals of real order. Int. J. Comput. Math., 31:125-135, 1990. 
[5] Chiccoli C., Lorenzutta S., Maino G. On a Tricomi series representation for the generalized exponential integral. Int. J. Comput. Math., 31:257-262, 1990.

[6] Chiccoli C., Lorenzutta S., Maino G. An algorithm for exponential integrals of real order. Computing, 45:269-276, 1990.

[7] DiDonato A.R., Morris Jr A.H. Computation of the incomplete gamma functions. ACM Trans. Math. Software, 12:377-393, 1986.

[8] Gautschi W. Exponential integral $\int_{1}^{\infty} e^{-x t} t^{-n} d t$ for large values of $n$. J. Res. Nat. Bur. Standards, 62:123-125, 1959.

[9] Gautschi W. Efficient computation of the complex error function. SIAM J. Numer. Anal., 7:187-198, 1970.

[10] Gautschi W. ALGO 363, The complex error function. Comm. ACM, 12:280, 1970.

[11] Gautschi W. A computational procedure for incomplete gamma functions. ACM Trans. Math. Software, 5:466-481, 1979. Algorithm 542, 482-489.

[12] Lozier D.W., Olver F.W.J. Numerical evaluation of special functions. In Proceedings of the Symposium on Mathematics of Computation, August 1993, Vancouver. AMS Proc. Symp. in Appl. Math, 1994. W. Gautschi, ed.

[13] Luke Y.L. The special functions and their approximations, Vols. I, II. Academic Press, New York, 1969.

[14] Olver F.W.J. Asymptotics and special functions. Academic Press, New York, 1974.

[15] Olver F.W.J. Uniform, exponentially improved, asymptotic expansions for the generalized exponential integral. SIAM J. Math. Anal., 22:1460-1474, 1991.

[16] Poppe G.P.M., Wijers C.M.J. More efficient computation of the complex error function. ACM Trans. Math. Software, 16:38-46, 1990.

[17] Temme N.M. On the computation of the incomplete gamma functions for large values of the parameters, pages 479-489. In Algorithms for approximation, Oxford, 1987. E.J.C. Mason and M.G. Cox, eds.

[18] Temme N.M. A set of algorithms for the incomplete gamma functions. To appear in Probability in the Engineering and Informational Sciences, 1994.

[19] Tricomi F.G. Asymptotische Eigenschaften der unvollständigen Gammafunktion. Math. Z., 53:136-148, 1950. 